\section{Molecular markers for prediction of risk of radiation- related injury to normal tissue}

\author{
Marco Ghilotti, ${ }^{1,2}$ Marco Alessandro \\ Pierotti, ${ }^{1,3}$ Manuela Gariboldi ${ }^{1,2}$ \\ 'IFOM, Fondazione Istituto FIRC di \\ Oncologia Molecolare, Milan, Italy; \\ 2Dept. of Experimental Oncology, \\ Fondazione IRCCS Istituto Nazionale dei \\ Tumori Milan, Italy; \\ ${ }^{3}$ Scientific Directorate, Fondazione IRCCS \\ Istituto Nazionale dei Tumori Milan, Italy
}

\section{Abstract}

Radiotherapy is one of the most effective methods for the treatment of cancer, but occurrence of adverse reactions developing in the co-irradiated normal tissue can be a threat for patients. Identification of individuals at risk of severe reaction is very difficult and considerable efforts have been made to correlate normal tissue toxicity with cellular responses to ionizing radiation. Genetic markers enabling to identify hyper-sensitive patients prior to treatment would considerably improve its outcome. Gene association studies should help to identify such markers. Expression levels of specific transcripts could be putative markers; in fact different studies found associations between gene expression profiles in normal cells and the reaction of normal tissues to radiation therapy. The finding that ionizing radiation induces the deregulation of a high number of genes suggests that also microRNAs that affect the expression of a large number of target genes may be involved. This review briefly introduces the mechanisms of radiationinduced normal tissue toxicity and summarizes clinical research focused on the evaluation of molecular biomarkers for predicting risk of injury to normal tissue, mainly describing gene transcripts alterations.

\section{Introduction}

Among non surgical treatments, ionizing radiation (IR) is the most effective therapy for local cancer control. It is an effective anti-cancer therapy commonly used to treat about 50 $60 \%$ of patient and aims at local tumor control (Radiotherapy WC Report 2003). However, in $5-10 \%$ of the cases it results in severe toxicity to normal tissues. ${ }^{1}$ Understanding the mechanisms responsible for IR toxicity and identifying at risk individuals before treatment decision would be helpful in individualizing and optimizing cancer treatment. Several studies aimed at finding biological predictors by looking at correlations between risk of radiationinduced injury and genomic or transcript variations. A biological marker identifying individuals at risk of radiation-induced injury would allow to perform patients stratification and to escalate dose accordingly, maximizing individual therapeutic gain.

\section{lonizing radiation toxicity to normal tissue}

IR therapy can cause two different types of toxicity: early, or acute toxicity, occurring during or within weeks of radiation exposure, and late toxicity that appears several months or even 1 year after the end of treatment (Figure 1). The clinical manifestations of both toxicities are well documented. Early effects can usually appear after radiotherapy to breast, lung and intestine and affect rapidly proliferating tissues, such as skin, gastrointestinal tract and the haematopoietic system. ${ }^{2}$ They include erythema, dry or moist desquamation of the skin, mucositis, nausea and diarrhoea. ${ }^{2}$ The occurrence of such a reaction is unpleasant for the patient, requires considerable care and significantly increases the risk of later development of skin conditions such as telangiectasia. In addition, if the reaction is very severe, it can necessitate an interruption in the scheduled treatment, while the available data suggest that a prolongation in treatment time may result in decreased local control. ${ }^{3,4}$ Late effects become manifest a long time after the end of treatment in prostate, breast, bone marrow and in some childhood cancers. Typically, they occur in more slowly proliferating tissues, such as kidney, heart and central nervous system, and trigger fibrosis, atrophy and vascular damage, which can lead to bleeding. ${ }^{25,56}$ As late side effects such as hormone deficiencies, infertility and second malignancies can be permanent, they provide the basis for dose constraints to radiation toxicity. ${ }^{7}$ Known risk factors include radiation dose and volume, conditions of the patient, concurrent chemotherapy, age and possible abnormalities in the genes involved in DNA repair mechanisms., ${ }^{3,8}$ Evidence has emerged that the development of radiation induced injury in normal tissue is influenced by patient-to-patient variability ${ }^{9}$ and there are increasing indications that genetic predisposition is a determining factor. ${ }^{10}$ This hypothesis finds support in the observed hyper radio-sensitivity associated with some rare autosomal recessive genetic diseases such as Ataxia Telangiectasia (A-T), ${ }^{11}$ AT-like disorder, ${ }^{12}$ Nijmegen breakage syndrome $^{13}$ and severe combined immuno-deficiencies. Patients carrying a single mutation in ATM gene, which is altered in AT patients, have a slightly increased risk of breast cancer, while specific sequence variants of $A T M$ may
Correspondence: Manuela Gariboldi, IFOM Fondazione Istituto FIRC di Oncologia Molecolare, via Adamello, 16, 20139 Milano, Italy. E-mail: manuela.gariboldi@ifom-ieo-campus.it and manuela.gariboldi@istitutotumori.mi.it

Key words: radiation, normal tissue toxicity, molecular markers.

Acknowledgements: the authors thank Dr. D. Majerna for help with editing the manuscript. This work was supported by grants from the Associazione Italiana per la ricerca sul Cancro (AIRC) and from Ministry of Health (Progetto Finalizzato Oncologia).

Contributions: MGA and MAP designed the structure of the review; MGH and MGA wrote the manuscript; MGA and MAP edited it; all the authors approved the final version for publication.

Conflict of interest: the authors report no conflicts of interest.

Received for publication: 15 September 2010. Accepted for publication: 5 October 2010.

This work is licensed under a Creative Commons Attribution 3.0 License (by-nc 3.0).

(C) Copyright M. Ghilotti et al., 2010

Licensee PAGEPress, Italy

Journal of Nucleic Acids Investigation 2010; 1:e11 doi:10.4081/jnai.2010.e11

predict for late adverse radiation responses. ${ }^{14}$ All the described disorders are very rare and of limited relevance in explaining the observed IR toxicity that has the characteristics of a complex polygenic trait resulting from the interaction of low-penetrance genetic variants (SNPs) of different genes with modest functional effects involved in diverse cellular pathways. ${ }^{15,16}$ The major challenge could be to identify the combination of multiple SNPs which affects the complex cellular and clinical phenotype of these patients and that could potentially serve as biomarkers predicting normal tissue response after IR. ${ }^{17,18}$

Expression of various genes belonging to IRregulated pathways (DNA repair, apoptosis, cell cycle control, fibrosis, cell adhesion, intracellular signalling, metabolism and stress response) is modulated in response to radiation. Starting from the hypothesis that differences in gene modulation could contribute to IR toxicity, the identification of the genes differentially expressed between patients who experience IR side effects and patients who do not could help in characterizing the response to radiotherapy. ${ }^{5,19}$ Some studies have found an association between gene expression profiles in normal cells, such as peripheral blood lymphocytes, and the reaction of normal tissues to 
radiation therapy. ${ }^{20}$ Other studies tested the possibility that, in sensitive patients, constitutive alterations in the expression levels of specific RNAs could be associated with IR toxicity, and identified genes with different expression both in the normal injured tissue and in peripheral blood. ${ }^{5,19,21-28}$ By assessing these genes at baseline, i.e. before the start of therapy, it could be possible to use them as biomarkers for predicting or monitoring normal tissue toxicity of radiation therapy, ${ }^{29}$ and thus select cases that can be directed towards a specific therapy, or change radiotherapy dosefractionation prescription or plan dose distributions. $^{30}$ Recently, various studies showed that IR can also modulate the expression levels of the microRNAs (miRNAs), ${ }^{31,32}$ short non coding RNAs (about 22 nucleotides in length) that are important regulators of gene expression..$^{33}$ The small size and resistance to RNase degradation make MiRNAs attractive diagnostic biomarkers, even superior to mRNAs(34). For these reasons they constitute a new class of promising IR biomarkers.

\section{SNPs associated with ionizing radi- ation toxicity}

SNPs association studies adopted the candidate gene approach, examining the genes involved in biological pathways that could determine the phenotype of interest. These genes include the cytokine TGF $\beta$ - 1 that can be activated by IR probably as a response to oxidative stress and inflammation, ${ }^{2}$ and gives rise to fibrosis $^{35,36}$ and other cytokines such as those from the cytocrome P450 family including CYP2D6 ${ }^{37}$ The 509 C>T SNP in TGF $\beta-1$ has been identified as a potential candidate biomarker for predicting the development of fibrosis after radiotherapy in breast and prostate cancer. $^{35,36}$ SNPs in ATM, have also been described; ${ }^{14,3841}$ for example, variant $5557 \mathrm{G}>\mathrm{A}$ has been associated with fibrosis and telangiectasia in breast cancer patients, ${ }^{42}$ while other specific variants have been found in radio-sensitive prostate cancer patients. ${ }^{41}$ SNPs related to other genes involved in DNA damage repair mechanisms, such as damage sensors, damage mediators, check point control, Non Homologous End Joining (NHEJ) repair and Homologous Recombination (HR), or in Base Excision Repair (BER) ${ }^{43,44}$ have been identified as well. However, none of them presents a strong association with IR sensitivity and so far it has not been possible to demonstrate a complete linkage of a specific genotype to IR toxicity. The multi-genic component of radiosensitivity cannot probably be investigated with a simple SNP based candidate gene approach; most likely each gene variant that contributes to sensitivity will have a low-penetrance effect and does not substantially contribute to the patient's clinical presentation after radiotherapy. Given the high frequency of

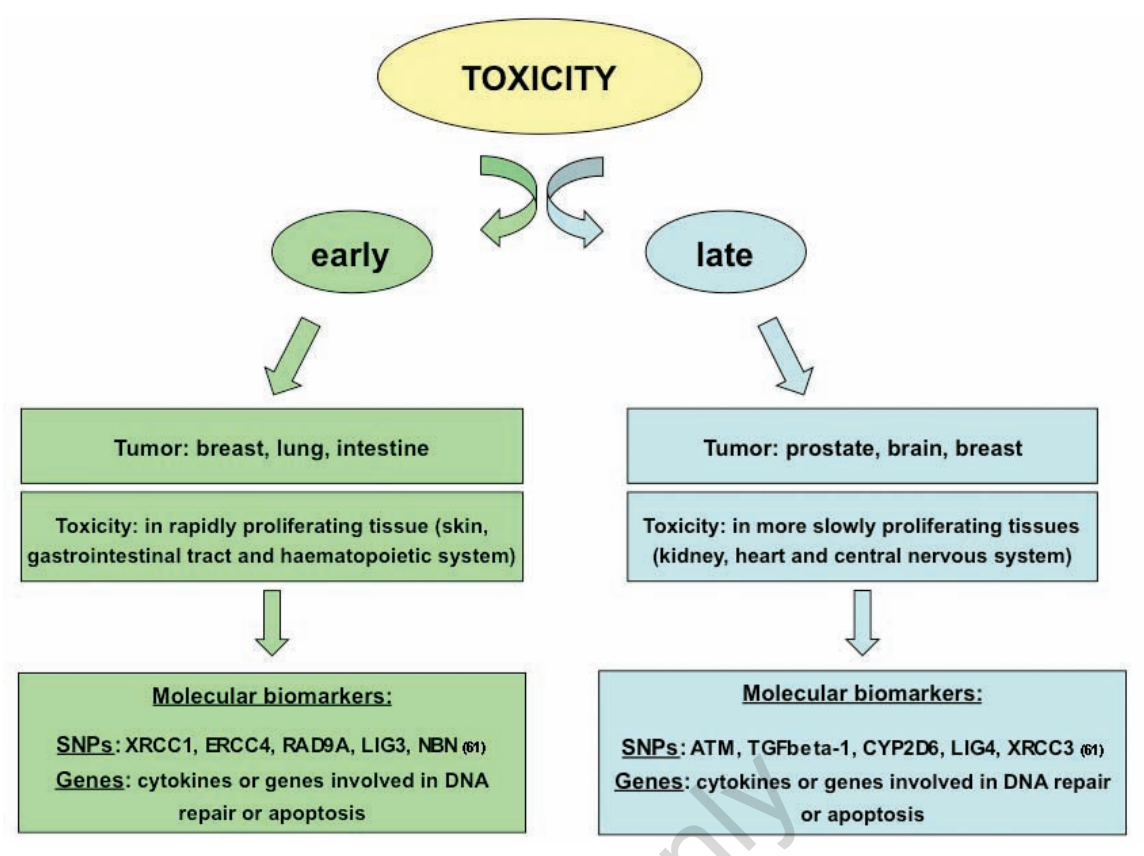

Figure 1. Toxicity of radiotherapy and possible biomarkers predictive of early or late events. SNPs in genes associated to IR-sensitivity quoted from Popanda et al. ${ }^{61}$

these gene variants, multiple SNPs, each identifying a specific gene variant, are probably required to affect the normal tissue response. For this reason, the application of whole genome scans (GWAS) with dense maps of SNPs that cover all the genome ${ }^{45}$ could be a promising approach. These association studies have already been successful in finding novel genetic variants that explain a useful proportion of the risk of developing some common cancers, ${ }^{46}$ even without prior knowledge of location or function. ${ }^{46-48}$

\section{Gene expression differences asso- ciated to ionizing radiation toxicity}

Based on the assumption that IR hypersensitivity may reflect inherited genetic defects associated with abnormal transcriptional responses to radiation, the investigation of differences in the transcriptional response by high through-put gene expression profiling may be a valid approach to identifying individuals at risk of side effects. ${ }^{49}$ In recent years, microarray technology has been increasingly used in the field of cancer research, and analyses of gene expression have been conducted on irradiated cells from cancer and normal tissue, or on lymphocytes (either stimulated or EBV-immortalized) in order to understand the side effects of IR toxicity. ${ }^{5,1921-28}$ The majority of the studies used RNA from biopsies in an attempt to identify prognostic classifiers. ${ }^{50}$ Many analyses have been conducted on normal tissues to understand their constitutive response to IR and possibly to identify distinctive genes for sensitive individuals that could be used as biomarkers for predicting radiosensitivity (Table 1).

The first studies on gene expression were conducted on single genes or on gene families such as cytokines (small glycoproteins involved in intercellular signaling) or growth factors involved in mediating IR toxicity. For example Li et al. demonstrated in a cohort of 91 early-stage breast cancer patients that TGF $\beta$-1 levels in pre-treatment plasma samples are related to subsequent development of radiation-induced breast fibrosis, ${ }^{21}$ while Chen et al. showed that prolonged cytokine expression post-radiotherapy is correlated to lung pneumonitis. ${ }^{51}$ Chaudhry et al. monitored radiation induced alterations on the expression of 6 genes including RGS1 (involved in the G-protein signalling pathway), CC3 (from the complement system), THBS1 (an extracellular matrix component), vWF (involved in blood coagulation), MADH7 (member of the TGF- $\beta$ signal transduction pathway), and on the expression of a transcript corresponding to a neuron derived neurotrophic factor (NENF) in four cell lines of different origin, Jurkat, TK6, HeLa, and HFL1.$^{27}$ All the analyzed genes were modulated by IR but in different manners, depending 0 the type of cell line, thus indicating a cell type specific involvement of different pathway in response to IR treatment.

Numerous studies where microarray analyses were used to compare baseline expression profiles from patients with severe versus mild 
normal tissue damage after radiotherapy have been conducted in the last few years. One of these studies analysed RNA from cultured fibroblasts obtained from 3 breast cancer patients with minimal or no fibrosis and three patients with severe post-radiotherapy fibrosis. Using a microarray enriched in cytokine RNAs, 9 cytokine-receptor transcripts were found significantly elevated in patients that had developed fibrosis. ${ }^{22}$ Another study on 5 head and neck cancer patients measured gene expression changes in peripheral blood mononucleated cells (PBMCs) before chemo-radiotherapy treatment and two-weeks after its start. ${ }^{25}$ Fourteen pathways, including genes belonging to inflammatory pathways like NFkB, IL-6 and VEGF signalling, were identified as being most deregulated in IR sensitive patients. These results demonstrated the validity of PBMCs as RNA source for genetic studies.

Other studies of gene expression have been performed on ex vivo models consisting in in vitro stimulated PBMCs, lymphoblastoid cell lines (LCLs) obtained from blood Epstein-Barr virus (EBV)-immortalized lymphocytes or cell lines derived from normal fibroblasts of at risk individuals and controls. ${ }^{5,19,23,24}$ The use of models enables to overcome the limitations of working with fresh blood samples and allows to keep cells under controlled growth condition, thus eliminating differences due to factors other than genetic (i.e. epigenetic or environmental factors). In addition, a number of established LCLs from patients affected by well known genetically inherited defects causing radiosensitive syndromes such as Ataxia Telangiectasia (A-T) can be used as positive controls to validate correlations of the transcriptional profile to radio-sensitivity. In the first study published on this subject, a gene expression profiling was performed to predict acute radiation toxicity on irradiated LCLs from 14 radiation-sensitive patients suffering from different types of cancer (breast cancer, Hodgkin's disease, low-grade lymphoma, cancer of tongue and salivary gland, endometrial cancer, orbital pseudo-tumor) and 43 controls with normal response to IR. ${ }^{23}$ After irradiation, a cluster of 24 genes mainly involved in DNA repair and apoptosis mechanisms was predictive of IR sensitivity. In a similar study, Svensson et al. used stimulated peripheral lymphocytes from 21 prostate cancer patients with severe late complications from radiation therapy and from 17 patients without symptoms. Cells were irradiated with $2 \mathrm{~Gy} \mathrm{X}$-rays and gene expression profiling was analyzed before and after irradiation. Irradiation induced the expression of numerous genes, and these were then used to develop a 72 genes signature predictive of late radiation toxicity that correctly classified $63 \%$ of the patient population in terms of whether or not the patient had developed toxicity. ${ }^{24}$ These two studies clearly demonstrated a relationship between gene expression profiles for lymphocytes irradiated ex vivo and the development of acute or late radiation injury to normal tissue.

Rødningen et al. profiled the fibroblast cell lines obtained from breast cancer patients with variable risk of radiation-induced fibrosis and found a set of 18 genes that could differentiate between high and low risk patients. MXRA5 (Matrix-remodeling associated 5) was the gene that best distinguished the 2 groups having a $>6$-fold higher expression level in high risk-samples. ${ }^{26}$

LCLs were also analyzed in a gene expression analysis conducted to investigate late side effects in a cohort of patients with prostate cancer. Valdagni et $a l .{ }^{5}$ tried to elucidate the reason why, despite excellent rectal dose-volume histograms (the DVH describes the cumulative distribution of dose over a specific volume) some patients experience rectal bleeding (LRB) while others, with poor DVHs, do not. Thirty-five genes involved in DNA repair/radiation response and apoptosis, part of which had already been found modulated by treatment in Rieger et al. ${ }^{23}$ were analyzed by quantitative real-time PCR in a cohort of patients enrolled in a clinical trial that investigated the correlation between LRB and dosimetric parameters. ${ }^{8}$ The study included 30 patients undergoing conformal radiotherapy with prescription doses higher than 70 Gy (minimum follow-up 48 months): 10 of them were selected among the low-risk or LRB cases (i.e. rectal DVH with the percent volume of rectum, $\mathrm{V}$, receiving

Table 1. Main studies aimed at identifying early and late toxicity in patients or in vitro. The table includes the principal author's name, the tumor or cell type used and the most significant findings.

\begin{tabular}{|c|c|c|c|}
\hline Early toxicity & Tumor type and tissue tested & Findings & Modulation \\
\hline Sonis S.25 & Head and Neck (PBMCs) 5 cases & IL-2, IL-6, TGF $\beta-1$, Bax, p53, TNF- $\alpha$ & Induced by IR \\
\hline Rieger K.E. ${ }^{23}$ & $\begin{array}{l}\text { Breast, Hodgkin's disease, lymphoma, } \\
\text { tongue and salivary gland cancer, endometrial } \\
\text { cancer, orbital pseudotumor } \\
\text { and brainstem (LCLs) } 14 \text { cases }\end{array}$ & $\begin{array}{l}24 \text { genes including NUDT1, RAD23B, RUVLB1, } \\
\text { CALM1, MAPKAP2, PPM1A, UBB, PSMB4, PSMD1, } \\
\text { CCNB1, CDC28, TNFSF7, SLC25A6, SLC25A5 }\end{array}$ & Induced by IR \\
\hline Henriquez Hernandez L.A. ${ }^{28}$ & Breast (LCLs) 12 cases & $\begin{array}{l}20 \text { genes including CCT8, SEC61G, SAMD3, GABARAP } \\
29 \text { genes including KRT17, IDH1, RPN2, EMP2, IQSEC1 }\end{array}$ & $\begin{array}{l}\text { Constitutive } \\
\text { Induced by IR }\end{array}$ \\
\hline Ghilotti M. ${ }^{19}$ & Breast (LCLs) 20 cases & H2AX mechanisms and chromatin structure & Constitutive \\
\hline Late toxicity & Tumor type and tissue tested & Findings & Modulation \\
\hline Li C. ${ }^{21}$ & Breast (plasma) 91 cases & TGF $\beta-1$ & Induced by IR \\
\hline Quarmby S.22 & Breast (fibroblasts) 6 cases & $\begin{array}{l}\text { FMLP, TNF } \alpha, N G F R, N T R K 1, E P H B 2, L F N G, \\
D D R 1, I F N G R, P D G F B\end{array}$ & Induced by IR \\
\hline Svensson J.P. ${ }^{24}$ & Prostate (PBMCs) 21 cases & 72 genes including CDKN1A, GADD45A, FAS, DDB2, XPC & Induced by IR \\
\hline Rødningen $0 . \mathrm{K}^{26}$ & Breast (fibroblasts) 31 cases & 18 genes including $M X R A 5$ & Induced by IR \\
\hline Valdagni R. ${ }^{5}$ & Prostate (LCLs + PBMCs) 30 cases & $\begin{array}{l}13 \text { genes including DRAP-1, LSM7, PSMB4 (LCLs) } \\
\text { DRAP-1 (PBMCs) }\end{array}$ & $\begin{array}{l}\text { Constitutive } \\
\text { Constitutive }\end{array}$ \\
\hline Henriquez Hernandez L.A..$^{28}$ & Breast (LCLs) 12 cases & $\begin{array}{l}26 \text { genes including DDA3, STXBP1, KIF20A, } \\
\text { RIT1, TPM2, C20orf155, DKFZP434L1 }\end{array}$ & Constitutive \\
\hline Model & Cell lines & Findings & Modulation \\
\hline Chaudrhy M.A. ${ }^{27}$ & $\begin{array}{l}\text { Jurkat (immortalized T lymphocytes) } \\
\text { TK6 (human lymphoblastoid normal cells) } \\
\text { Hela (human epithelia cervical cancer) } \\
\text { HFL1 (human lung embryonic) }\end{array}$ & RGS1, CC3, THBS1, vWF, MADH7, NENF & Induced by IR \\
\hline
\end{tabular}


more than $70 \mathrm{~Gy}<20 \%$ and the percent volume of rectum receiving more than $50 \mathrm{~Gy}<55 \%$ with Grade 2 or Grade 3, G2-G3 toxicity) and 20 among patients classified as at high-risk of bleeding or HRB (V70Gy > 25\% and V50Gy $>60 \%$, with G2-G3 toxicity), 10 of which had side effects (i.e. rectal bleeding, HRB) and 10 that had no toxicity (high risk not bleeding, HRNB). Intergroup comparison (between patients with low DVH and patients with high DVH) showed many constitutive differences: nine genes were significantly down-regulated in the LRB group with respect to the two high risk groups (HRB+HRNB): AKR1B, BAZ1B, LSM7, MRPL23, NUDT1, PSMB4, PSMD1, SEC22L1 and $U B B$, all with a $\mathrm{P}<0.05$. Four genes were significantly up-regulated in the HRNB group: DDX17, DRAP1, RAD23 and SRF, all with $\mathrm{P}<0.05$. LSM7 and PSMB4 were the best predictors of enhanced radio-sensitivity, while enhanced radio-resistance was best predicted by DRAP-1. Twenty-seven genes resulted IR-regulated in at least one group, bleeders having (LRB+HRB) almost twice the numbers of modulated genes than HRNB. The constitutive difference of DRAP-1 was also confirmed in stimulated PBMCs obtained from the same cases $(\mathrm{P}=0.03)$. These results, if validated in larger case series, propose DRAP-1 as a possible biomarker that could be used in clinical practice to identify patients to whom more "flexible" DVH constraints and/or higher RT doses could be administered safely.

Henriquéz-Hernández et al. studied early and late toxicity by profiling un-irradiated and irradiated LCLs obtained from breast cancer patients that had either early or late (after 6 months of follow-up) toxicity. They obtained a group of 81 genes regulated by radiotherapy and found 20 and 26 constitutive genes associated with acute and late toxicity, respectively. After irradiation, 29 genes were found associated with early toxicity while none was related to the development of late toxicity. ${ }^{28}$

Ghilotti et al. tried to identify markers useful for predicting early radiation sensitivity in women with breast cancer that showed acute side effects after radiotherapy. ${ }^{19}$ They analysed LCLs derived from PBMCs of 10 women who manifested high toxicity after radiotherapy (grade G2 and G4) and of 10 women which had no side effects (G0). LCLs were used to investigate the molecular mechanisms underlying the differences in clinical radio-sensitivity, as a first step towards the identification of markers to stratify patients according to treatment reaction. Sensitive patients showed a different capacity of repairing double strand breaks induced by IR, which was measured by phosphorylation of histone $\mathrm{H} 2 \mathrm{AX}$, and presented a more compact chromatin. A further approach for identifying possible markers to predict radio-sensitivity was to search IR induced alterations in the expression levels of genes that, together with the histone $\mathrm{H} 2 \mathrm{AX}$, constitute the foci, or are directly involved in DSBs repair. Genes found associated with late toxicity in patients with prostate cancer ${ }^{5}$ were also analyzed. A significant modulation in expression levels among the 2 groups (radio-resistant and radio-sensitive patients) was observed, but there were no genes whose different expression could predict radiation toxicity. ${ }^{19}$ If the data obtained from in vitro analyses of LCLs could be confirmed in larger cohorts of samples and in vivo, predictive tests to detect the effect of early toxicity induced by radiotherapy in patients with breast cancer could be proposed for clinical practice.

The above summarized studies make a novel contribution to the clarification of the relationship between the constitutive gene expression profile of peripheral blood lymphocytes and toxicity after IR treatment. They open up the possibility that the different constitutive expression levels of a selected group of genes may predict acute and late toxicity.

\section{MicroRNAs and response to radiation}

The complexity of genetic cellular response to radiation highlighted by microarray studies suggests that miRNAs, which are potential regulators of the expression levels of a large number of target genes, may be required to influence the radiation response. MiRNAs have been studied as potential diagnostic or therapeutic targets in cancer treatment and an asso- ciation between miRNA expression in tumors and radio-sensitivity has been observed ${ }^{52}$ (Table 2). The role of miRNAs in the DNA damage response (DDR) pathway is just emerging and some miRNAs appear to modulate the response to cytotoxic therapy through regulation of DDR genes. ${ }^{53}$ MiRNA expression profiles have been correlated with sensitivity or resistance to certain chemotherapeutic agents. ${ }^{54}$ Several studies demonstrated that miR-34 family is a direct target of TP53 and mediates some of the TP53-dependent effects including DNA damage repair. ${ }^{55}$ Kato et al. used the Caenorhabditis elegans model to determine the role of miR-34 in radiation-induced cell death in vivo and found an abnormal cellular survival response to radiation when miR-34 is mutationally inactivated. The authors assessed the radio-sensitivity both of a normal breast epithelial line (HMEC) with high levels of miR-34 and of a breast cancer cell line (MDAMB-231) that had low expression of miR-34, and found that MDA-MB-231 cells were significantly more radiosensitive. Transfection of miR-34 into MDA-MB-231 cells protected them from radiation- induced cell death. ${ }^{56}$ These findings confirm that miR-34 is required for a normal cellular response to DNA damage in vivo resulting in altered cellular survival postirradiation and point to a potential therapeutic use for anti-miR-34 as a radio-sensitizing agent in p53-mutant breast cancers. ${ }^{56}$

To understand the basic genetic principles

Table 2. In vitro and in vivo MicroRNAs modulation after ionising radiation.

\begin{tabular}{|c|c|c|c|}
\hline Reference & Cell lines or tissue & MicroRNA & Modulation \\
\hline Kato $\mathrm{M}^{56}$ & $\begin{array}{l}\text { HMEC (human mammary epithelial cell) } \\
\text { MDA-MB-231 (breast cancer cell line) }\end{array}$ & miR-34 & Induced \\
\hline Weidhaas J.B. ${ }^{53}$ & A549 (lung cancer cell line) & $\begin{array}{l}\text { Let-7 family } \\
\text { (except Let-7g) } \\
\text { Let-7g }\end{array}$ & $\begin{array}{l}\text { Down-modulated } \\
\text { Induced }\end{array}$ \\
\hline Josson $S^{59}$ & LNCaP, C4-2 (prostate cancer cell lines) & $\begin{array}{l}\text { miR-34 } \\
\text { miR-133 } \\
\text { miR-196 } \\
\text { miR-521 }\end{array}$ & $\begin{array}{l}\text { Induced } \\
\text { Down-modulated }\end{array}$ \\
\hline Chaudhry M.A. ${ }^{60}$ & $\begin{array}{l}\text { TK6 (human lymphoblastoid normal cell) } \\
\text { WTK-1 (human lymphoblastoid p53 mutant cell) }\end{array}$ & $\begin{array}{l}\text { miR-15 } \\
\text { miR-16 } \\
\text { miR-21 } \\
\text { Let-7 family }\end{array}$ & $\begin{array}{l}\text { Up in TK6 (0.5Gy) } \\
\text { Down in WTK-1 (0.5Gy) } \\
\text { Down in TK6 (2Gy) } \\
\text { Down in TK6 (0.5Gy) } \\
\text { Up in TK6 (2Gy) } \\
\text { Up in TK6 } \\
\text { Down in WTK-1 }\end{array}$ \\
\hline Wagner-Ecker M. ${ }^{32}$ & $\begin{array}{l}\text { HDMEC (human dermal microvascular } \\
\text { endothelial cells) }\end{array}$ & $\begin{array}{l}\text { miR-16 } \\
\text { miR-20a } \\
\text { miR-21 } \\
\text { miR-29c } \\
\text { Let-7g } \\
\text { miR-18a } \\
\text { miR-125a } \\
\text { miR-127 } \\
\text { miR-148b } \\
\text { miR-189 } \\
\text { miR-503 }\end{array}$ & Down-modulated \\
\hline Koturbash I.57 & Rat (plasma and spleen) & miR-194 & Induced \\
\hline
\end{tabular}


involved in irradiation Koturbash et al. monitored the role of epigenetic changes in the development of secondary malignancies in near-by non-irradiated tissue during radiotherapy in rats. ${ }^{57}$ After whole body or cranial irradiation, alterations in DNA methylation, histone methylation and miRNA expression were investigated in spleen and blood. From 24 hours up to 7 months after irradiation, a significant loss of global DNA methylation and down-regulation of DNA methyltransferases and MeCP2 (methyl-binding protein methyl CpG binding protein 2), the key regulators of DNA methylation, were observed. At the same time points, miRNA profiling highlighted elevated levels of miR-194, which putatively targets both DNA methyltransferase-3a and MeCP2. Overall, miR-194 seems to play some role in the maintenance of the long-term response. ${ }^{58}$

Experiments with cancer cell lines in therapeutic radio-therapy settings revealed that irradiation causes a wide range of alterations in miRNA expression during therapy. Weidhass et al. compared miRNA profiles of a lung cancer cell line, A549, before and after irradiation and found that levels of 81 out of the 440 miRNAs analysed differed significantly. Part of them was also IR modulated in normal lung epithelium cells suggesting that a highly conserved global miRNA response exists in lung after irradiation. Among the modulated miRNAs, all the 8 members of the Let-7 family except Let-7g decreased significantly by $2-8$ hours after irradiation in both cancerous and normal lung epithelium. Transfection of A549 cells with members of the let-7 family increased radiosensitivity whereas decreasing their levels induces radioresistance in an in vivo model of radiation-induced cell death in Caenorhabditis elegans, partly through the control of the proto-oncogene homologue let-60/RAS and of genes in the DNA damage response pathway. These findings are the first direct evidence that miRNAs can suppress resistance to anticancer cytotoxic therapy, a common feature of cancer cells, and suggest that miRNAs may be a viable tool to augment current cancer therapies. ${ }^{53}$

Josson et al. analysed the modulation of 330 miRNAs in 2 radiosensitive prostate cancer cell lines, LNCaP and $\mathrm{C} 4-2$, and found that almost half of them were deregulated by treatment. Some of these miRNAs were common to both cell lines, for example miR-521, miR-196 and miR-133, which decreased, and miR-34, which was induced by irradiation. Introduction of miR-521 (the most prominently down-regulated miRNA identified) in LNCaP cells made them more sensitive to radiation treatment, while its inhibition determined an increase of resistance to IR treatment, proposing miR-521 as modulator of radiation response..$^{59}$

Chaudhry et al. investigated by quantitative real-time PCR the role of miRNAs in IR response in 2 human cell lines, TK6 and WTK1, that differed in p53 status and radiation sensitivity, to verify if alterations in p53 determine changes in miRNA responses to IR..$^{60}$ Without IR exposure the 2 cell lines already showed differences in miRNA expression. After irradiation with either 0.5 or $2 \mathrm{~Gy}$ doses of X-rays, many miRNAs markedly differed within the same cell line. In particular, the expression of miRNAs from the let-7 family was up-regulated in irradiated TK6 cells but down-regulated in WTK1 cells. MiR-15a and miR-16 were up-regulated in 0.5Gy-irradiated TK6 cells but downregulated after a 2Gy dose of X-rays. Expression of the same miRNAs decreased in 0.5Gy-exposed WTK1. MiR-21 was up-regulated in 0.5Gy-treated TK6 cells and its target genes PDCD4, PTEN and SPRY2 were found to be down-regulated. MiR-21 was down-regulated in 2Gy-irradiated TK6 cells, while PDCD4, PTEN and SPRY2 were up-regulated in 2Gyexposed TK6 cells. These results confirm a direct involvement of miRNAs in IR response and their dependence from $\mathrm{p} 53$ status.

Wagner-Ecker et al. studied IR effects on human endothelial cells (HDMEC) and found that radiation up-regulates the expression of let-7g, miR-16, miR-20a, miR-21 and miR-29c, and reduces the expression of miR-18a, miR125a, miR-127, miR-148b, miR-189 and miR503. Over-expression or inhibition of let-7g, miR-20a and miR-189 markedly influenced clonogenic survival and cell proliferation. Radio-sensitivity of HDMEC was significantly influenced by differential expression of miR125a, miR-127, miR-189, and let-7g: while miR$125 \mathrm{a}$ and miR-189 had a radio-protective effect, miR-127 and let-7g enhanced radio-sensitivity. ${ }^{32}$

In summary, miRNA expression is either down- or up-modulated by radiation treatment in different models and can be under control of genes regulating response to irradiation such as p53. Modulation of some miRNAs, for example miR-15, miR-16 or miR-21, depends on the dose of radiation or on the tissue type, suggesting that miRNAs act at specific steps of response to irradiation. All these findings suggest that miRNAs could be promising biomarkers for a direct blood test from patients before radiotherapy to determine differences in radiation sensitivity. In addition, some miRNAs could be targeted for therapeutic benefit, for example anti-miR-34 molecules might prove useful in radio-sensitizing tumors for better treatment.

\section{Conclusions}

Although the literature on biomarkers in cancer biology and tumor therapy outcome is rapidly expanding, the study of biomarkers in normal tissue radiobiology is still at the begin- ning. A number of studies have reported mainly positive associations between certain genetic variants and the risk of injury to normal tissue after radiotherapy, supporting the hypothesis that normal tissue toxicity can be considered as a complex quantitative trait and that naturally occurring genetic variations can to some extent account for the observed interpatient variability. GWASs will probably enable to capture an additional fraction of the existing genetic determinants. Gene and miRNA expression analyses will complement this investigation. Gene expression profiles have identified a number of genes that can discriminate at risk patients, and in some cases patients classification has been improved by considering the joint behavior of functionally related genes belonging to the same pathways. This approach might better accommodate the existing genetic heterogeneity within a patient group. The joint behavior of functionally or spatially related genes may be significant, whereas the activity of individual genes may not. Another advantage of this approach is that it might lead to a more relevant biological interpretation of the results. However, no single gene or functionally related set of genes was found that, by itself, correlated perfectly with the observed clinical radiation toxicity. Several studies identified miRNAs that were altered in response to radiation treatment. Functional studies showed that miRNAs can confer radiation sensitivity by modulating DDR proteins, making them promising markers for predicting IR sensitivity. The studies summarized here support the hypothesis that patients who develop severe reactions to radiotherapy have an intrinsic radiosensitivity that can be identified in peripheral blood lymphocytes by quantifying gene expression response to IR.

Most of the prognostic profiles discussed in the review have been tested on one dataset and the predictive assays have only been validated on small numbers of samples. A larger, possibly multicentric validation is needed, and the biological mechanisms in which these markers are involved should be investigated to support the rationale for their use in clinical settings. The final goal will be to establish the basis for a simple clinical test that could be used to predict response to treatment therefore enabling to stratify patients according to their risk-level and adjust radiation to individualize patient treatment.

\section{References}

1. Radiotherapy, in: B.W. Stewart, P. Kleihues (Eds), World Cncer Report, IARC Press, International Agency for Research on 
Cancer (IARC), World Health Organisation (WHO), Lyon, 2003;277-280.

2. Bentzen SM. Preventing or reducing late side effects of radiation therapy: radiobiology meets molecular pathology. Nat Rev Cancer 2006;6:702-13.

3. Back M, Guerrieri M, Wratten C, Steigler A. Impact of radiation therapy on acute toxicity in breast conservation therapy for early breast cancer. Clin Oncol ( $\mathrm{R}$ Coll Radiol) 2004;16:12-6.

4. Fisher J, Scott C, Stevens R, et al. Randomized phase III study comparing Best Supportive Care to Biafine as a prophylactic agent for radiation-induced skin toxicity for women undergoing breast irradiation: Radiation Therapy Oncology Group (RTOG) 97-13. Int J Radiat Oncol Biol Phys 2000;48:1307-10.

5. Valdagni R, Rancati T, Ghilotti M, et al. To bleed or not to bleed. A prediction based on individual gene profiling combined with dose-volume histogram shapes in prostate cancer patients undergoing three-dimensional conformal radiation therapy. Int $\mathrm{J}$ Radiat Oncol Biol Phys 2009;74:1431-40.

6. Bolling T, Schuck A, Pape H, et al. German register for detection of late sequelae after radiotherapy for children and adolescents (RiSK): present status and first results. Strahlenther Onkol 2007;183:7-8.

7. Barnett GC, West CM, Dunning AM, et al. Normal tissue reactions to radiotherapy: towards tailoring treatment dose by genotype. Nat Rev Cancer 2009;9:134-42.

8. Fiorino C, Sanguineti G, Cozzarini C, et al. Rectal dose-volume constraints in highdose radiotherapy of localized prostate cancer. Int J Radiat Oncol Biol Phys 2003;57:953-62.

9. Andreassen CN. Can risk of radiotherapyinduced normal tissue complications be predicted from genetic profiles? Acta Oncol 2005;44:801-15.

10. Gatti RA. The inherited basis of human radiosensitivity. Acta Oncol 2001;40:70211.

11. Savitsky K, Bar-Shira A, Gilad S, et al. A single ataxia telangiectasia gene with a product similar to PI-3 kinase. Science 1995;268:1749-53.

12. Stewart GS, Maser RS, Stankovic T, et al. The DNA double-strand break repair gene hMRE11 is mutated in individuals with an ataxia-telangiectasia-like disorder. Cell 1999;99:577-87.

13. Varon R, Vissinga C, Platzer M, et al. Nibrin, a novel DNA double-strand break repair protein, is mutated in Nijmegen breakage syndrome. Cell 1998;93:467-76.

14. Ho AY, Fan G, Atencio DP, et al. Possession of ATM sequence variants as predictor for late normal tissue responses in breast cancer patients treated with radiotherapy. Int
J Radiat Oncol Biol Phys 2007;69:677-84.

15. Andreassen CN, Alsner J, Overgaard J. Does variability in normal tissue reactions after radiotherapy have a genetic basis-where and how to look for it? Radiother Oncol 2002;64:131-40.

16. Travis EL. Genetic susceptibility to late normal tissue injury. Semin Radiat Oncol 2007;17:149-55.

17. Andreassen CN, Alsner J, Overgaard M, Overgaard J. Prediction of normal tissue radiosensitivity from polymorphisms in candidate genes. Radiother Oncol 2003; 69:127-35.

18. Bartsch H, Dally H, Popanda O, et al. Genetic risk profiles for cancer susceptibility and therapy response. Recent Results Cancer Res 2007;174:19-36.

19. Ghilotti M, Lozza L, Fontanella E, et al. Constitutive differences in DNA repair and chromatin compaction identify breast cancer patients susceptible to acute radiation toxicity (Submitted).

20. Nuyten DS, van de Vijver MJ. Using microarray analysis as a prognostic and predictive tool in oncology: focus on breast cancer and normal tissue toxicity. Semin Radiat Oncol 2008;18:105-14.

21. Li C, Wilson PB, Levine E, et al. TGF-beta1 levels in pre-treatment plasma identify breast cancer patients at risk of developing post-radiotherapy fibrosis. Int J Cancer 1999;84:155-9.

22. Quarmby S, West C, Magee B, et al. Differential expression of cytokine genes in fibroblasts derived from skin biopsies of patients who developed minimal or severe normal tissue damage after radiotherapy. Radiat Res 2002;157:243-8.

23. Rieger KE, Hong WJ, Tusher VG, et al. Toxicity from radiation therapy associated with abnormal transcriptional responses to DNA damage. Proc Natl Acad Sci U S A 2004;101:6635-40.

24. Svensson JP, Stalpers LJ, Esveldt-van Lange RE, et al. Analysis of gene expression using gene sets discriminates cancer patients with and without late radiation toxicity. PLoS Med 2006;3:e422.

25. Sonis S, Haddad R, Posner M, et al. Gene expression changes in peripheral blood cells provide insight into the biological mechanisms associated with regimenrelated toxicities in patients being treated for head and neck cancers. Oral Oncol 2007;43:289-300.

26. Rodningen OK, Borresen-Dale AL, Alsner $\mathrm{J}$, et al. Radiation-induced gene expression in human subcutaneous fibroblasts is predictive of radiation-induced fibrosis. Radiother Oncol 2008;86:314-20.

27. Chaudhry MA. Analysis of gene expression in normal and cancer cells exposed to gamma-radiation. J Biomed Biotechnol
2008;541678.

28. Henriquez Hernandez LA, Lara PC, Pinar $\mathrm{B}$, et al. Constitutive gene expression profile segregates toxicity in locally advanced breast cancer patients treated with highdose hyperfractionated radical radiotherapy. Radiat Oncol 2009;4:17.

29. Bentzen SM, Parliament M, Deasy J0, et al. Biomarkers and surrogate endpoints for normal-tissue effects of radiation therapy: the importance of dose-volume effects. Int J Radiat Oncol Biol Phys 2010; 76:S145-50.

30. Bentzen SM. From cellular to highthroughput predictive assays in radiation oncology: challenges and opportunities. Semin Radiat Oncol 2008;18:75-88.

31. Cha HJ, Seong KM, Bae S, et al. Identification of specific microRNAs responding to low and high dose gammairradiation in the human lymphoblast line IM9. Oncol Rep 2009;22:863-8.

32. Wagner-Ecker M, Schwager C, Wirkner U, et al. MicroRNA expression after ionizing radiation in human endothelial cells. Radiat Oncol 2010;5:25.

33. Lim LP, Lau NC, Garrett-Engele P, et al. Microarray analysis shows that some microRNAs downregulate large numbers of target mRNAs. Nature 2005;433:769-73.

34. Waldman SA, Terzic A. Translating MicroRNA discovery into clinical biomarkers in cancer. JAMA 2007;297:1923-5.

35. Feltl D, Zavadova E, Pala M, Hozak P. Posttreatment plasma transforming growth factor beta 1 (TGF-beta1) level predicts for late morbidity in patients with advanced head and neck cancer. Neoplasma 2005;52:393-7.

36. Zhao L, Sheldon K, Chen M, et al. The predictive role of plasma TGF-betal during radiation therapy for radiation-induced lung toxicity deserves further study in patients with non-small cell lung cancer. Lung Cancer 2008;232-9.

37. Damaraju S, Murray D, Dufour J, et al. Association of DNA repair and steroid metabolism gene polymorphisms with clinical late toxicity in patients treated with conformal radiotherapy for prostate cancer. Clin Cancer Res 2006;12:2545-54.

38. Edvardsen H, Tefre T, Jansen L, et al. Linkage disequilibrium pattern of the ATM gene in breast cancer patients and controls; association of SNPs and haplotypes to radio-sensitivity and post-lumpectomy local recurrence. Radiat Oncol 2007;2:25.

39. Iannuzzi CM, Atencio DP, Green S, et al. ATM mutations in female breast cancer patients predict for an increase in radiation-induced late effects. Int $\mathrm{J}$ Radiat Oncol Biol Phys 2002;52:606-13.

40. Bremer M, Klopper K, Yamini P, et al. Clinical radiosensitivity in breast cancer 
patients carrying pathogenic ATM gene mutations: no observation of increased radiation-induced acute or late effects. Radiother Oncol 2003;69:155-60.

41. Cesaretti JA, Stock RG, Lehrer S, et al. ATM sequence variants are predictive of adverse radiotherapy response among patients treated for prostate cancer. Int $\mathbf{J}$ Radiat Oncol Biol Phys 2005;61:196-202.

42. Angele S, Romestaing P, Moullan N, et al. ATM haplotypes and cellular response to DNA damage: association with breast cancer risk and clinical radiosensitivity. Cancer Res. 2003;63:8717-25.

43. O'Driscoll M, Jeggo PA. The role of doublestrand break repair - insights from human genetics. Nat Rev Genet 2006;7:45-54.

44. Petrini JH, Stracker TH. The cellular response to DNA double-strand breaks: defining the sensors and mediators. Trends Cell Biol 2003;13:458-62.

45. Kruglyak L. The road to genome-wide association studies. Nat Rev Genet 2008;9:3148.

46. Easton DF, Eeles RA. Genome-wide association studies in cancer. Hum Mol Genet. 2008;17:R109-15.

47. Thomas DC, Haile RW, Duggan D. Recent developments in genomewide association scans: a workshop summary and review. Am J Hum Genet 2005;77:337-45.

48. Wellcome Trust Case Control Consortium.
Genome-wide association study of 14,000 cases of seven common diseases and 3,000 shared controls. Nature 2007;447:661-78.

49. Morley M, Molony CM, Weber TM, et al. Genetic analysis of genome-wide variation in human gene expression. Nature 2004;430:743-7.

50. Kruse JJ, Stewart FA. Gene expression arrays as a tool to unravel mechanisms of normal tissue radiation injury and prediction of response. World $\mathrm{J}$ Gastroenterol 2007;13:2669-74.

51. Chen Y, Hyrien 0, Williams J, et al. Interleukin (IL)-1A and IL-6: applications to the predictive diagnostic testing of radiation pneumonitis. Int J Radiat Oncol Biol Phys 2005;62:260-6.

52. Hummel R, Hussey DJ, Haier J. MicroRNAs: predictors and modifiers of chemo- and radiotherapy in different tumour types. Eur J Cancer 2010;46:298311.

53. Weidhaas JB, Babar I, Nallur SM, et al. MicroRNAs as potential agents to alter resistance to cytotoxic anticancer therapy. Cancer Res 2007;67:11111-6.

54. Salter KH, Acharya CR, et al. An integrated approach to the prediction of chemotherapeutic response in patients with breast cancer. PLoS One 2008;3:e1908.

55. He L, He X, Lim LP, et al. A microRNA component of the p53 tumour suppressor net- work. Nature 2007;447:1130-4.

56. Kato M, Paranjape T, Muller RU, et al. The mir-34 microRNA is required for the DNA damage response in vivo in C. elegans and in vitro in human breast cancer cells. Oncogene 2009;28:2419-24.

57. Koturbash I, Boyko A, Rodriguez-Juarez R, et al. Role of epigenetic effectors in maintenance of the long-term persistent bystander effect in spleen in vivo. Carcinogenesis 2007;28:1831-8.

58. Ilnytskyy Y, Koturbash I, Kovalchuk 0. Radiation-induced bystander effects in vivo are epigenetically regulated in a tissue-specific manner. Environ Mol Mutagen 2009;50:105-13.

59. Josson S, Sung SY, Lao K, et al. Radiation modulation of microRNA in prostate cancer cell lines. Prostate 2008;68:1599-606.

60. Chaudhry MA, Kreger B, Omaruddin RA. Transcriptional modulation of micro-RNA in human cells differing in radiation sensitivity Int J Radiat Biol 2010;86:569-83.

61. Popanda 0, Tan XL, Ambrosone CB, et al. Genetic polymorphisms in the DNA double-strand break repair genes XRCC3, XRCC2, and NBS1 are not associated with acute side effects of radiotherapy in breast cancer patients. Cancer Epidemiol Biomarkers Prev 2006;15:1048-50. 